\title{
Falls-Related Drug Use and Risk of Falls Among Older Adults: A Study in a US Medicare Population
}

\author{
Shirley Musich ${ }^{1} \cdot$ Shaohung S. Wang ${ }^{1}$ Joann Ruiz ${ }^{2} \cdot$ Kevin Hawkins $^{1}$ • \\ Ellen Wicker ${ }^{3}$
}

Published online: 3 June 2017

(c) The Author(s) 2017. This article is an open access publication

\begin{abstract}
Background Approximately one-third of communitydwelling older adults fall each year, and approximately $10 \%$ have falls requiring medical services. Among other factors, research studies have linked certain medications with an increased risk of falls.

Objective The aim of this study was to examine the risk of falls relative to use patterns among new and continuing falls-related drug (FRD) users.

Methods A $10 \%$ random sample, insured in AARP $^{\circledR}$ Medicare Supplement and AARP Medicare Part D Rx plans, was utilized to define new and continuing FRD users. New users had a 12-month pre-period without FRD use, whereas continuing users had at least one FRD in the pre-period. Both groups had 12-month follow-up after initiating or continuing FRD use. Characteristics associated with the risk of falls for new and continuing users were determined using multivariate logistic regression models.
\end{abstract}

Shirley Musich

shirley.musich@optum.com

Shaohung S. Wang

sara.wang@optum.com

Joann Ruiz

joann_ruiz@uhc.com

Kevin Hawkins

kevin.hawkins@optum.com

Ellen Wicker

ewicker@aarp.org

1 Advanced Analytics, Optum, 315 E. Eisenhower Parkway, Suite 305, Ann Arbor, MI 48108, USA

2 Medicare and Retirement, UnitedHealthcare Alliances, PO Box 9472, Minneapolis, MN 55440, USA

3601 E. Street, N.W., Washington, DC 20049, USA
Results Among insureds, $44 \%$ used at least one of the FRD classes. Of these, $29 \%$ were new users $(N=35,340)$ and $71 \%$ were continuing users $(N=121,488)$. Fall rates for the two subgroups were similar at 7 and $8 \%$, respectively. Characteristics associated with the risk of falls were previous injurious fall, use of two or more classes of FRDs, older age, poorer health, and being female. New users were at higher risk than continuing users.

Conclusion New users of FRDs were at highest risk of falls, and continuing users were at increased risk, especially with higher numbers of FRD classes. Both groups could benefit from falls awareness and prevention programs.

\section{Key Points}

This study of AARP Medicare Supplement insureds was designed to explore the potential of a proactive falls intervention focused on the use patterns of fallsrelated drugs (FRDs) and subsequent risk of falling.

New users of multiple classes of FRDs were at the highest risk for falls; however, continuing users remained at higher risk for falls, especially with higher numbers of FRD classes.

Falls awareness and prevention programs would be feasible, targeting those using two or more classes of FRDs among new and continuing FRD users. 


\section{Introduction}

Approximately one-third of community-dwelling older adults fall each year, and approximately $10 \%$ have falls resulting in non-fatal injuries that require medical services $[1,2]$. Generally, risk factors for falling include being older, female, lower level of education, lower income, having depression or dementia, unsteady gait, poorer health, and previous injurious falls [2-5]. Research studies have also linked certain medications with an increased risk of falls or hip fractures among older adults, including antidepressants, antihypertensives, diuretics, some analgesics, sedative hypnotics, and psychotropic medications. Although research studies have associated these selected falls-related drugs (FRDs) with the risk of falls, there is no comprehensive, evidence-based list of specific problematic medications in general use. European researchers use a list of fall-risk increasing drugs [6-13]; the US Veterans Administration has used a list from the Canadian Safety Council [14, 15]; other US researchers have utilized an augmented list recommended by Woolcott et al. [16-18]; and others have created FRD lists from various published research studies [19].

Often, research study designs assess FRD utilization before and after an injurious fall event or hospitalization for a hip fracture [7-9, 18]. This approach is intended to serve secondary prevention purposes such as preventing a second fall in the future. However, general trends for FRD use tend to be higher after the event than before despite efforts to reduce prescriptions of these drugs. In alignment with this strategy, the US National Committee for Quality Assurance (NCQA) has defined a Healthcare Effectiveness Data and Information Set (HEDIS) measure that addresses 'potentially harmful drug-disease interactions in the elderly' as follows [20]:

Elderly patients who had an accidental fall or hip fracture who took an anticonvulsant, non-benzodiazepine hypnotic, selective serotonin reuptake inhibitor (SSRI), antiemetic, antipsychotic, benzodiazepine, or tricyclic antidepressant after the incident.

For the assessment of this quality measure, HEDIS has defined a recommended list of FRD classes. The goal of the measure is to monitor and reduce the numbers of prescriptions of these specific FRDs for patients who have experienced an injurious fall. However, a more proactive strategy focused on primary prevention might target the FRD users prior to their fall. Such a strategy might include the development of intervention programs that could reduce the number of falls in targeted at-risk populations.
The association of the individual HEDIS-defined FRD classes with falls has been well established [3, 14, 15, 17, 21, 22]. Generally, the associations have been documented in research designs that assess the individual FRD classes independently [11, 15, 22]. Fewer studies have focused on combinations of FRD classes $[6,12,14,19]$. In these studies, higher numbers of FRD classes [6], specifically two or more classes [12, 14, 19], were associated with an increased incidence of falls or hip fractures.

In addition, while the increased risk of a fall associated with initiating a new prescription of one specific class of FRDs, i.e. benzodiazepines, has been documented compared to the risk associated with longer-term users, few studies have incorporated this information into their study designs [23-25]. Specifically, the risk of a hip fracture was highest in the first 2 weeks after initiating a new prescription for a benzodiazepine. The risk then linearly decreased in the second 2 weeks and then again after 1 month of continued use of the drug [23]. The relationship of increased risk of a fall associated with new FRD users compared with continuing users has apparently not been tested with other classes of FRDs.

Interventions to address the risk of falls associated with FRDs have primarily focused on medication reviews by nurses, pharmacists, research physicians, or hospital physicians $[7-10,13,18,26,27]$. These programs attempt to reduce the number of FRD prescriptions by withdrawing patients completely from selected FRDs, or at least reducing doses. While this approach has demonstrated some success (i.e. reducing prescriptions and/or falls) in small-scale research studies, most attempts have generally concluded that deprescribing drugs for older adults has proven difficult. In one recent intervention focused on patients with injurious falls, $40 \%$ of study patients could not have their drug regimens altered, and another $35 \%$ refused to change for a variety of reasons [13]. Furthermore, among those who changed by reducing FRDs, $47 \%$ resumed the drugs 6 months after the intervention concluded [27]. Nevertheless, when patients reduced their FRD prescriptions, their risk of falls was also reduced [10, 27].

Our approach for this study was to determine if we could define at-risk subgroups of older adults suitable for a targeted, proactive, falls awareness and prevention program. Thus, the focus of the HEDIS quality measure was reversed to assess patterns of FRD utilization that might prospectively put individuals at increased risk for a fall. We found no published research studies to date investigating the association of FRDs with the risk of falls among older adults with Medicare Supplement plans (i.e. Medigap) $[18,23]$. In the US, government-funded Medicare covers adults aged 65 years and older, as well as those under 65 years of age and disabled. Medicare fee-for-service 
plans (approximately $70 \%$ of all Medicare plans) pay approximately $80 \%$ of medical expenditures for these individuals but offer no prescription drug benefits. Those enrolled in these Medicare plans are personally responsible for obtaining additional insurance plans to cover the remaining $20 \%$ of medical expenses (i.e. Medicare Supplement or Medigap plans) and prescription drug coverage (Medicare Part D Rx plans). While most (approximately $90 \%$ ) of those with original fee-for-service Medicare coverage have some type of supplemental insurance coverage, approximately $28 \%$ (currently approximately 10.2 million adults) have purchased Medigap coverage [28]. Since this population may differ from general older adult and/or specifically Medicare populations, it was of interest to determine the risk of falls or hip fractures associated with selected patterns of utilization (i.e. use of multiple classes) for FRDs among these older adults and to detail associated characteristics for those who had a fall compared with those who did not experience a fall.

This study adds to the research literature on FRDs, with its focus on a Medigap study population, and in considering the prospective risk of falls among new and continuing FRD users by categories of multiple FRD classes. While some studies have focused on risks associated with multiple classes of FRDs, none have included both aspects of risk of falls: (1) stratification by new and continuing users, and (2) impact of taking multiple classes of these drugs.

Thus, our primary objective was to estimate the risk of falls relative to utilization patterns for new and continuing FRD users among AARP ${ }^{\circledR}$ Medicare Supplement insureds. Characteristics associated with new and continuing FRD users who had a fall were determined and were utilized in considering the feasibility of a targeted, prospective, falls prevention intervention. FRD utilization patterns focused on the relative impact on the risk of falls associated with increasing multiple FRD classes. This research was covered under the New England IRB No. 120160532.

\section{Methods}

\subsection{Sample Selection}

In 2015, approximately 4 million Medicare insureds were covered by an AARP Medicare Supplement plan insured by UnitedHealthcare Insurance Company (for New York residents, UnitedHealthcare Insurance Company of New York). These plans are offered in all 50 states, Washington DC, and various US territories. A $10 \%$ random sample of 2015 AARP Medicare Supplement insureds with AARP Medicare Part D Rx plans (approximately $55 \%$ of insureds have AARP Part D plans) was utilized to define new and continuing FRD users. New users of FRDs had a variable date range 12-month pre-period (1 August 2013-31 July 2015) without any FRD use, and had a variable date range 12-month follow-up after initiating FRD use (1 August 2015-31 July 2016). Meanwhile, continuing users had at least one FRD prescription in the pre-period (1 January 2014-31 December 2014) and filled at least one prescription of FRD in 2015 (1 January 2015-31 December 2015). Individuals in both groups must have been at least 64 years of age at study initiation. The final study populations that met the inclusion criteria included 35,340 new users and 121,148 continuing users.

\subsection{Healthcare Effectiveness Data and Information Set (HEDIS) Falls-Related Drug (FRD) Classes}

Since there is no consistency in the FRDs included in research studies, we chose to use the FRDs sourced from HEDIS-specified FRD classes with associated National Drug Category (NDC) codes [20]. This set of FRDs included the following drug classes: anticonvulsants, nonbenzodiazepine hypnotics, selective serotonin reuptake inhibitors (SSRIs), antiemetics, antipsychotics, benzodiazepines, and tricyclic antidepressants. The number of FRD classes was counted and categorized as one class, two classes, three classes, and four or more classes. Individuals were identified by the highest number of FRD prescriptions during the 12-month follow-up period, with no minimum number of months taking a specific drug.

\subsection{Falls}

The primary condition of interest for this study was the incidence of falls or hip fractures in the 12-month time period following initiation of at least one FRD (new users) or during the calendar year of 2015 (continuing users). Falls requiring medical services or hip fractures, as a combined measure, were defined from suggested HEDIS diagnosis codes [20]. In addition, any injurious fall in the 12-month pre-period was included in the regression models as an additional control associated with the known risk for a second fall given a first event [2]. Falls were documented as any fall (single or multiple) during the 12-month followup period for new and continuing FRD users compared with the respective FRD users without an event. Since these analyses were designed to determine feasibility of a prospective falls intervention, it was more important to document if an individual had a fall given FRD use (i.e. odds ratios [ORs]) than to assess the timing of that event relative to drug initiation (i.e. hazard ratios) [5, 22]. 


\subsection{Covariates}

Covariates were included to characterize individuals who experienced a fall that required medical services and to adjust for other risk factors associated with the likelihood for this event. These covariates included measures of demographics, socioeconomic factors, health status and other characteristics taken from health plan eligibility and administrative medical claims.

Demographic questions included age and sex. Age groups were defined as 64-69, 70-79, and $\geq 80$ years. Geographic regions (Northeast, South, Midwest and West) and low, medium, and high minority areas were geocoded from zip codes. AARP Medicare Supplement plan types were grouped by cost-sharing levels, including high-level coverage plans with minimal copayments or deductibles (plans C, F, and J), medium-level coverage (plans B, D, E, $\mathrm{G}, \mathrm{H}, \mathrm{I}$, and $\mathrm{N}$ ), and all other plans (plans A, K, L, and nonstandardized plans). Access to care was measured as the number of acute care hospital beds per 100,000 in the individual's hospital service area. Level of medical services utilization from medical claims was calculated as the Hierarchical Condition Category (HCC) score [29], which is used by the Centers for Medicare \& Medicaid Services (CMS) to risk-adjust medical payments across various medical plans according to the health status of the different insured populations. The HCC score in the pre-period was utilized as a continuous variable in regression models as a control for health status. Prescribers associated with specific FRD prescriptions were identified from pharmacy claims and, subsequently, the number of unique prescribers for FRD prescriptions per patient was determined: one prescriber, two prescribers, three prescribers, or four or more prescribers.

\subsection{Prevalence of Common Chronic Conditions}

Eleven medical conditions used in calculating the Charlson Comorbidity Index (CCI) score [30] were defined from diagnosis codes in the healthcare claims: acquired immunodeficiency syndrome/human immunodeficiency virus (AIDS/HIV), any malignancy, chronic obstructive pulmonary disease (COPD), dementia, diabetes (mild, moderate, or with chronic complications), heart problems (myocardial infarction, heart failure, peripheral vascular disease), liver disease (mild, moderate, or severe), peptic ulcer disease, renal disease, rheumatologic disease, or stroke. The CCI is a measure of the risk of 1-year all-cause mortality attributable to selected comorbidities that has also been shown to be highly predictive of morbidity and healthcare expenditures [30]. These medical conditions were utilized in regression models to additionally control for health status in the pre-period. Those conditions that correlated strongly with the HCC score were dropped from the models.

\subsection{Statistical Models}

Characteristics associated with those who experienced a fall compared with those who did not fall, among new and continuing users of FRDs, were determined using multivariate logistic regression models. Covariates included all of those variables listed in Table 1. Variables with high correlations (e.g., $>0.5$ ) were dropped from the models. All analyses were completed using SAS Enterprise Guide Version 6.1 (SAS Institute Inc., Cary, NC, USA).

\section{Results}

Overall, among the $10 \%$ random sample of AARP Medicare Supplement insureds with AARP Medicare Part D Rx plans $(N=447,169)$, approximately $44 \%(N=200,175)$ were using at least one FRD. Of these, approximately $28 \%$ $(N=56,625)$ were new FRD users and approximately $72 \%$ $(N=143,550)$ were continuing FRD users. After study inclusion criteria were applied, the final study populations included 35,340 new (62\%) and 121,148 continuing (84\%) FRD users. Fall rates for the two groups were similar, i.e. $7 \%(N=2532)$ and $8 \%(N=9738)$, respectively, compared with rates of approximately $4 \%$ among non-users of these drugs (data not shown). New and continuing users were mostly female (63 and 69\%, respectively), 70-79 years of age (47 and $47 \%$, respectively), White (49 and $49 \%$ low minority, respectively), and living in the South (40 and 42\%, respectively). The most common FRD classes among new users were benzodiazepines (46\%), anticonvulsants (29\%), SSRIs (24\%), and non-benzodiazepine hypnotics (12\%). The most common FRD classes among continuing users were benzodiazepines (46\%), SSRIs (44\%), anticonvulsants (33\%), and non-benzodiazepine hypnotics $(17 \%)$ (Tables 1,2$)$.

\subsection{Characteristics Associated with Falls Among New and Continuing FRD Users}

Characteristics associated with new FRD users who had a fall included previous injurious fall, taking multiple classes of FRD drug classes (i.e. taking four or more classes, more than three classes, and more than two classes), older age, dementia, poorer health (higher HCC score), and being female. The two strongest predictors of having a fall among new FRD users were a previous fall (OR 3.85) and taking four or more FRD classes (OR 3.26) (Table 3; Fig. 1a). 
Table 1 Unadjusted demographics for new users of FRDs

\begin{tabular}{|c|c|c|c|c|}
\hline Variable & $\begin{array}{l}\text { Overall } \\
{[N=35,340]} \\
(\% \text { or mean })\end{array}$ & $\begin{array}{l}\text { Had a fall } \\
{[N=2532]} \\
(\% \text { or mean })\end{array}$ & $\begin{array}{l}\text { No fall } \\
{[N=32,808]} \\
(\% \text { or mean })\end{array}$ & $p$ value \\
\hline \multicolumn{5}{|l|}{ Sex } \\
\hline Male & 36.4 & 31.9 & 36.7 & \multirow[t]{2}{*}{0.0001} \\
\hline Female & 62.7 & 67.6 & 62.3 & \\
\hline Age, years & 75.9 & 80.5 & 75.5 & 0.0001 \\
\hline $64-69$ & 23.7 & 11.6 & 24.7 & 0.0001 \\
\hline $70-79$ & 47.1 & 33.8 & 48.1 & \\
\hline$\geq 80$ & 29.2 & 54.7 & 27.3 & \\
\hline \multicolumn{5}{|l|}{ Minority (from zip codes) } \\
\hline Low & 48.8 & 50.0 & 48.7 & \multirow[t]{3}{*}{0.04} \\
\hline Medium & 46.2 & 46.2 & 46.3 & \\
\hline High & 3.2 & 2.4 & 3.3 & \\
\hline \multicolumn{5}{|l|}{ Region (from zip codes) } \\
\hline Midwest & 14.8 & 18.1 & 14.6 & \multirow[t]{4}{*}{0.0001} \\
\hline Northeast & 24.3 & 24.9 & 24.2 & \\
\hline South & 40.1 & 38.6 & 40.2 & \\
\hline West & 20.3 & 18.4 & 20.5 & \\
\hline \multicolumn{5}{|l|}{ Plan type } \\
\hline High coverage & 78.2 & 74.7 & 78.5 & \multirow[t]{3}{*}{0.0001} \\
\hline Medium coverage & 2.9 & 2.9 & 2.9 & \\
\hline Other & 18.9 & 22.4 & 18.7 & \\
\hline Acute hospital beds $/ 100,000$ & 229.7 & 229.8 & 229.7 & 0.96 \\
\hline \multicolumn{5}{|l|}{ HCC score in pre-period } \\
\hline Pre-period & 1.15 & 1.60 & 1.11 & 0.0001 \\
\hline Post-period & 1.42 & 2.26 & 1.35 & 0.0001 \\
\hline \multicolumn{5}{|l|}{ CCI conditions in pre-period } \\
\hline AIDS/HIV & 0.1 & 0.2 & 0.0 & 0.001 \\
\hline Any malignancy & 17.9 & 18.4 & 17.9 & 0.51 \\
\hline COPD & 22.5 & 27.2 & 22.2 & 0.0001 \\
\hline Dementia & 3.4 & 9.6 & 3.0 & 0.0001 \\
\hline Diabetes & 24.8 & 26.0 & 24.7 & 0.15 \\
\hline Heart problems & 25.8 & 38.5 & 24.8 & 0.0001 \\
\hline Liver disease & 4.4 & 4.8 & 4.3 & 0.30 \\
\hline Peptic ulcer disease & 1.4 & 2.0 & 1.3 & 0.003 \\
\hline Renal disease & 10.3 & 15.4 & 9.9 & 0.0001 \\
\hline Rheumatologic disease & 4.7 & 6.1 & 4.6 & 0.001 \\
\hline Stroke & 16.7 & 26.1 & 16.0 & 0.0001 \\
\hline Injurious fall in pre-period & 5.6 & 21.8 & 4.3 & 0.0001 \\
\hline Filled two or more FRD classes & 24.9 & 37.3 & 23.9 & 0.0001 \\
\hline One class & 75.1 & 62.7 & 76.1 & 0.0001 \\
\hline Two classes & 19.5 & 26.3 & 19.0 & \\
\hline Three classes & 4.3 & 8.3 & 4.0 & \\
\hline Four or more classes & 1.0 & 2.7 & 0.9 & \\
\hline Multiple prescribers of FRDs & 29.4 & 44.4 & 28.3 & 0.0001 \\
\hline 1 prescriber & 70.6 & 55.7 & 71.7 & 0.0001 \\
\hline 2 prescribers & 20.6 & 26.0 & 20.2 & \\
\hline 3 prescribers & 6.0 & 11.3 & 5.6 & \\
\hline$\geq 4$ prescribers & 2.8 & 7.0 & 2.5 & \\
\hline
\end{tabular}


Table 1 continued

\begin{tabular}{|c|c|c|c|c|}
\hline Variable & $\begin{array}{l}\text { Overall } \\
{[N=35,340]} \\
(\% \text { or mean })\end{array}$ & $\begin{array}{l}\text { Had a fall } \\
{[N=2532]} \\
(\% \text { or mean })\end{array}$ & $\begin{array}{l}\text { No fall } \\
{[N=32,808]} \\
(\% \text { or mean })\end{array}$ & $p$ value \\
\hline \multicolumn{5}{|l|}{ FRDs } \\
\hline Anticonvulsants & 29.4 & 34.2 & 29.1 & 0.0001 \\
\hline Antiemetics & 10.9 & 10.5 & 11.0 & 0.46 \\
\hline Antipsychotics & 4.4 & 11.6 & 3.8 & 0.0001 \\
\hline Benzodiazepines & 46.2 & 44.6 & 46.3 & 0.09 \\
\hline Non-benzodiazepine hypnotics & 12.0 & 10.9 & 12.1 & 0.09 \\
\hline SSRIs & 23.7 & 34.8 & 22.8 & 0.0001 \\
\hline Tricyclic antidepressants & 4.8 & 4.9 & 4.8 & 0.81 \\
\hline
\end{tabular}

FRD falls-related drug, $H C C$ Hierarchical Condition Category, $C C I$ Charlson Comorbidity Index, $C O P D$ chronic obstructive pulmonary disease, AIDS/HIV acquired immunodeficiency syndrome/human immunodeficiency virus, SSRI selective serotonin reuptake inhibitor

Similarly, characteristics associated with continuing FRD users who had a fall included previous injurious fall or hip fracture, older age, taking multiple classes of FRD drug classes (i.e. taking four or more classes, more than three classes, and more than two classes), dementia, being female, and poorer health (higher HCC score). The two strongest predictors of having a fall among continuing FRD users were a previous fall (OR 3.38) and being $\geq 80$ years of age (OR 2.51). While continuing FRD users had similar risk factors for falls or hip fractures, the magnitude of the impact for most risk factors was lower compared with new users of these drugs (Table 4; Fig. 1b)

\subsection{Targeting a Suitable At-Risk Population for a Prospective Intervention}

Figure $1 \mathrm{a}, \mathrm{b}$ display the increase in magnitude of risk for a fall as the number of FRD classes increased for both new (Fig. 1a) and continuing (Fig. 1b) FRD users, along with the numbers of individuals within each category. The doseresponse relationship in fall risk linearly increased as additional FRD classes were added. The numbers of individuals in the highest risk categories (four or more classes), for example for both new and continuing users $(N=354$ and $N=3511$, respectively) provide suitably sized, at-risk subgroups that could be targeted for prospective falls interventions.

\section{Discussion}

In our population of AARP Medicare Supplement insureds, 44\% had used at least one class of FRDs. Of these, 29\% were new users and $71 \%$ were continuing users. Fall rates for the two groups were 7 and $8 \%$, respectively, similar to previously reported rates for injurious falls [2]. Higher reported fall rates (e.g. 30\%) are generally self-reported and include non-injurious falls, and thus were not included in this study [1].

Characteristics associated with the risk of falls or hip fractures were similar for new and continuing users: previous injurious fall, use of two or more classes of FRDs, older age, poorer health, and being female. These characteristics are in general agreement with other studies that have assessed risk factors associated with falls or hip fractures $[3,5,6,11,22]$. The strongest predictor for a fall event in our study was a previous injurious fall. These results are consistent with a previous, prospective, 5-year study demonstrating that individuals who experienced a previous injurious fall were at significantly higher risk of a second injurious fall in the future [2].

We stratified our population by new and continuing FRD use and by increasing numbers of FRD classes to demonstrate prospective risk of falls over a 1-year time period. To our knowledge, no previous studies have tested the risk of falls fractures for both new and continuing users of combined FRD classes. There was a dose response impact of increased fall risk with increased numbers of FRD classes. While both groups were at risk, new users were at a higher magnitude of risk for most risk factors compared with continuing users. However, continuing users were also at increased risk, especially with higher numbers of FRD classes (e.g., four or more classes). These results are in agreement with the benzodiazepine studies documenting that the risk of falls or hip fractures decreased over time with continued use of one specific FRD [23-25].

In addition, increased numbers of prescribers were also evident with increased numbers of FRD . Approximately $30-40 \%$ of those receiving FRDs had two or more prescribers, with $25-40 \%$ receiving two or 
Table 2 Unadjusted demographics for continuing users of FRDs

\begin{tabular}{|c|c|c|c|c|}
\hline Variable & $\begin{array}{l}\text { Overall }[N=121,148] \\
(\% \text { or mean })\end{array}$ & $\begin{array}{l}\text { Had a fall }[N=9738] \\
(\% \text { or mean })\end{array}$ & $\begin{array}{l}\text { No fall }[N=111,410] \\
(\% \text { or mean) }\end{array}$ & $p$ value \\
\hline \multicolumn{5}{|l|}{ Sex } \\
\hline Male & 30.0 & 23.6 & 30.5 & \multirow[t]{2}{*}{0.0001} \\
\hline Female & 69.0 & 75.6 & 68.5 & \\
\hline Age, years & 76.1 & 79.8 & 75.7 & 0.0001 \\
\hline $64-69$ & 22.9 & 12.4 & 23.8 & 0.0001 \\
\hline $70-79$ & 46.9 & 37.1 & 47.8 & \\
\hline$\geq 80$ & 30.1 & 50.5 & 28.4 & \\
\hline \multicolumn{5}{|l|}{ Minority (from zip codes) } \\
\hline Low & 48.8 & 48.9 & 48.8 & \multirow[t]{3}{*}{0.48} \\
\hline Medium & 46.9 & 47.1 & 46.8 & \\
\hline High & 2.9 & 2.6 & 2.9 & \\
\hline \multicolumn{5}{|l|}{ Region (from zip codes) } \\
\hline Midwest & 15.3 & 17.5 & 15.1 & \multirow[t]{4}{*}{0.0001} \\
\hline Northeast & 22.9 & 24.1 & 22.8 & \\
\hline South & 41.9 & 40.0 & 42.1 & \\
\hline West & 19.5 & 18.2 & 19.6 & \\
\hline \multicolumn{5}{|l|}{ Plan type } \\
\hline High coverage & 79.1 & 75.1 & 79.4 & \multirow[t]{3}{*}{0.0001} \\
\hline Medium coverage & 2.6 & 2.7 & 2.5 & \\
\hline Other & 18.4 & 22.2 & 18.1 & \\
\hline Acute care hospital beds $/ 100,000$ & 231.1 & 233.1 & 231.0 & 0.006 \\
\hline \multicolumn{5}{|l|}{ HCC score in pre-period } \\
\hline Pre-period & 1.27 & 1.69 & 1.23 & 0.0001 \\
\hline Post-period & 1.39 & 2.15 & 1.32 & 0.0001 \\
\hline \multicolumn{5}{|l|}{ CCI conditions in pre-period } \\
\hline AIDS/HIV & 0.1 & 0.0 & 0.1 & 0.16 \\
\hline Any malignancy & 15.9 & 15.9 & 15.9 & 0.93 \\
\hline COPD & 25.3 & 31.2 & 24.8 & 0.0001 \\
\hline Dementia & 4.8 & 11.7 & 4.2 & 0.0001 \\
\hline Diabetes & 26.1 & 27.8 & 26.0 & 0.0001 \\
\hline Heart problems & 27.0 & 40.9 & 25.8 & 0.0001 \\
\hline Liver disease & 4.3 & 4.3 & 4.4 & 0.95 \\
\hline Peptic ulcer disease & 1.5 & 1.9 & 1.5 & 0.001 \\
\hline Renal disease & 11.0 & 15.5 & 10.6 & 0.0001 \\
\hline Rheumatologic disease & 5.4 & 7.1 & 5.3 & 0.0001 \\
\hline Stroke & 18.1 & 26.1 & 17.4 & 0.0001 \\
\hline Injurious fall in pre-period & 6.7 & 22.5 & 5.3 & 0.0001 \\
\hline Filled more or two FRD classes & 42.4 & 53.4 & 41.4 & 0.0001 \\
\hline One class & 57.6 & 46.6 & 58.6 & 0.0001 \\
\hline Two classes & 29.5 & 33.0 & 29.2 & \\
\hline Three classes & 10.0 & 15.1 & 9.5 & \\
\hline More or four classes & 2.9 & 5.3 & 2.7 & \\
\hline Multiple prescribers of FRDs & 38.1 & 51.2 & 37.0 & 0.0001 \\
\hline 1 prescriber & 61.9 & 48.8 & 63.0 & 0.0001 \\
\hline 2 prescribers & 25.6 & 29.7 & 25.2 & \\
\hline 3 prescribers & 8.4 & 12.9 & 8.1 & \\
\hline$\geq 4$ prescribers & 4.1 & 8.7 & 3.7 & \\
\hline FRDs & & & & \\
\hline
\end{tabular}


Table 2 continued

\begin{tabular}{lcccr}
\hline Variable & $\begin{array}{l}\text { Overall }[N=121,148] \\
(\% \text { or mean })\end{array}$ & $\begin{array}{l}\text { Had a fall }[N=9738] \\
(\% \text { or mean })\end{array}$ & $\begin{array}{l}\text { No fall }[N=111,410] \\
(\% \text { or mean })\end{array}$ \\
\hline Anticonvulsants & 33.0 & 39.9 & 32.4 & 0.0001 \\
Antiemetics & 4.6 & 5.7 & 4.5 & 0.0001 \\
Antipsychotics & 6.8 & 13.1 & 6.3 & 0.0001 \\
Benzodiazepines & 45.7 & 46.8 & 45.6 & 0.02 \\
Non-benzodiazepine hypnotics & 16.9 & 14.1 & 47.2 & 0.0001 \\
SSRIs & 44.0 & 53.1 & 43.2 & 0.0001 \\
Tricyclic antidepressants & 7.6 & 7.6 & 7.7 \\
\hline
\end{tabular}

FRD falls-related drug, $H C C$ Hierarchical Condition Category, $C C I$ Charlson Comorbidity Index, COPD chronic obstructive pulmonary disease, AIDS/HIV acquired immunodeficiency syndrome/human immunodeficiency virus, SSRI selective serotonin reuptake inhibitor

Table 3 Characteristics associated with falls among new users of FRDs

\begin{tabular}{llc}
\hline Variable & New FRD users & \\
\cline { 2 - 3 } & Odds ratio for falls & $p$ value \\
\hline Injurious fall in pre-period & 3.85 & $<0.0001$ \\
Filled four or more FRD & 3.26 & $<0.0001$ \\
$\quad$ classes & & \\
Age $\geq 80$ years & 3.03 & $<0.0001$ \\
Filled three FRD classes & 2.14 & $<0.0001$ \\
Filled two FRD classes & 1.57 & $<0.0001$ \\
Age 70-79 years & 1.38 & $<0.0001$ \\
Dementia in pre-period & 1.37 & 0.0002 \\
HCC score in pre-period & 1.24 & $<0.0001$ \\
Midwest & 1.24 & 0.0006 \\
Female & 1.22 & $<0.0001$ \\
RD in pre-period & 1.06 & 0.56 \\
White & 1.05 & 0.26 \\
Plan type $=$ other & 1.03 & 0.62 \\
Northeast & 1.02 & 0.70 \\
Liver disease in pre-period & 1.02 & 0.88 \\
Acute hospital beds/100,000 & 1.00 & 0.16 \\
West & 0.99 & 0.85 \\
Plan type $=$ medium coverage & 0.95 & 0.70 \\
\hline
\end{tabular}

FRD falls-related drug, $H C C$ Hierarchical Condition Category, $R D$ rheumatologic disease

more FRD . According to the NCQA, on average Medicare beneficiaries utilize seven physicians a year, with six of these being specialists [31]. Care coordination across physicians to manage FRD drug prescriptions may be beneficial to older patients, although, to date, no studies have tested the effectiveness of this approach.

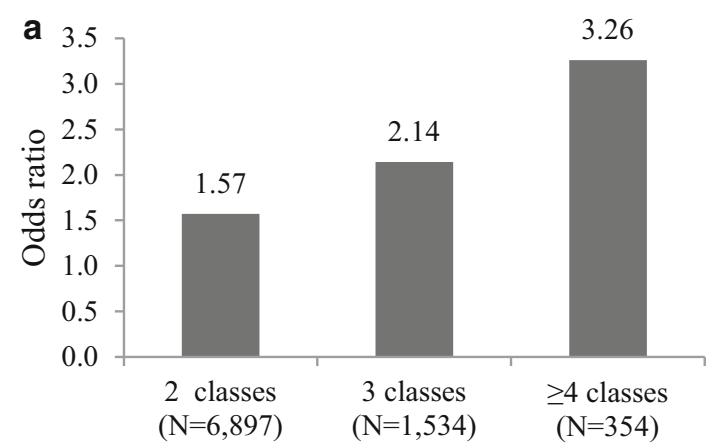

Number of drug classes

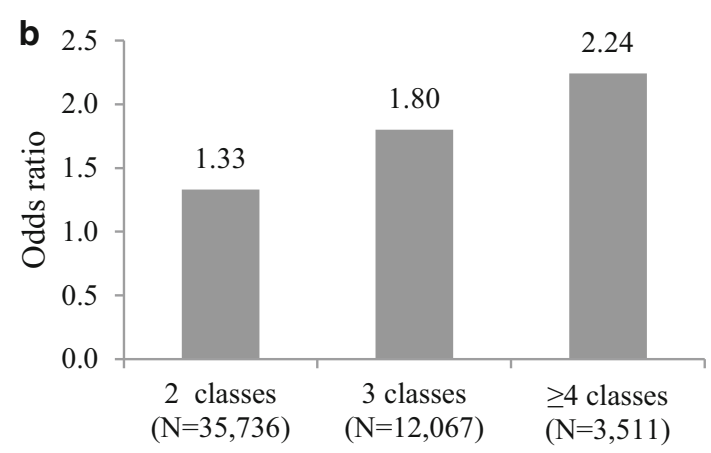

Number of drug classes

Fig. 1 Risk of falls among a new and $\mathbf{b}$ continuing FRD users. Both new and continuing FRD users were at increased risk of a fall associated with multiple FRD classes. New users were at a higher magnitude of risk. Numbers of individuals at risk by number of drug classes would indicate that an intervention could feasibly target those individuals at highest risk (four or more classes and/or three classes; new $>$ continuing users). FRD falls-related drug

Many interventions designed to address the fall risk association with FRD prescriptions have been based on medication reviews as a single-focus program to reduce falls. However, medication reviews, whether conducted by 
Table 4 Characteristics associated with falls among continuing users of FRDs

\begin{tabular}{llc}
\hline Variable & \multicolumn{2}{l}{ Continuing FRD users } \\
\cline { 2 - 3 } & Odds ratio for falls & $p$ value \\
\hline Injurious fall in pre-period & 3.38 & $<0.0001$ \\
Age $\geq 80$ years & 2.51 & $<0.0001$ \\
Filled four or more FRD & 2.24 & $<0.0001$ \\
$\quad$ classes & & $<0.0001$ \\
Filled 3 FRD classes & 1.80 & $<0.0001$ \\
Dementia in pre-period & 1.43 & $<0.0001$ \\
Age 70-79 years & 1.38 & $<0.0001$ \\
Filled 2 FRD classes & 1.33 & $<0.0001$ \\
Female & 1.32 & $<0.0001$ \\
HCC score in pre-period & 1.23 & $<0.0001$ \\
Midwest & 1.15 & 0.02 \\
RD in pre-period & 1.11 & 0.19 \\
Plan type $=$ medium coverage & 1.09 & 0.002 \\
Plan type = other & 1.09 & 0.05 \\
West & 1.06 & 0.06 \\
Northeast & 1.06 & 0.99 \\
White & 1.00 & 0.09 \\
Acute hospital beds/100,000 & 1.00 & 0.12 \\
Liver disease in pre-period & 0.92 & \\
\hline
\end{tabular}

$F R D$ falls-related drug, $H C C$ Hierarchical Condition Category, $R D$ rheumatologic disease

nurses, pharmacists or physicians, have demonstrated limited success in changing drug protocols [7-10, 26, 27]. Conditions often treated with FRDs include anxiety, depression, insomnia, behavioral problems (i.e., dementia patients), seizures, and panic disorders. Without addressing these underlying problems, it has proven difficult to keep patients off FRDs, even after they may have discontinued their prescriptions or have reduced their dosages [27]. Monitoring symptoms, including dizziness, gait instability, drowsiness, or decreased reflexes, may provide a mechanism to motivate change by both the physician and the patient, especially for new FRD users [5, 6, 22]. Likewise, deprescribing FRDs paired with cognitive behavioral therapy, support groups, or physician support has shown some signs of success $[17,21]$.

In a recent qualitative study, general practitioners did not generally perceive FRDs as a prominent factor regarding falls for their elderly patients, and expressed hesitation in altering drug protocols, especially among patients who had used their drugs for a relatively long period of time [32]. Other factors that inhibited change in drug use patterns included reluctance by the patient to discontinue use of drugs that have alleviated symptoms, multiple prescribers treating different conditions/ symptoms, and patients who may not have necessarily shared their symptoms with physicians [32].

In addition to medication reviews, other fall intervention approaches have included falls awareness, assessment, and prevention strategies. Multifactorial falls interventions have been shown to be effective in some studies but ineffective in others [33]. Fall prevention programs typically address physical issues (e.g., shoes, home hazards), psychological well-being (e.g., depression, anxiety), and symptom assessments (e.g., dizziness, drowsiness). Exercise programs have successfully targeted strength, balance, flexibility, and endurance in reducing the incidence of falls [33-35]. An innovative falls awareness and prevention intervention will likely require the incorporation of several of these aspects to reduce falls rates.

Regardless of the challenges of intervention design, this analysis has demonstrated that prospectively stratifying an older adult population by FRD utilization patterns can successfully identify suitable at-risk subgroups from administrative databases (Fig. 1a, b). To further support recommendations of this approach, administrative pharmacy claims databases are updated in near real-time in contrast to lag times of several months typically associated with medical claims. Thus, individuals could be targeted as soon as they add new FRD prescriptions, or when the count of FRD exceeds two or more.

Our study has some limitations. The study population of AARP Medicare Supplement insureds may not generalize to all older adults or other Medicare or Medicare Supplement beneficiaries. Injurious falls were documented from medical diagnosis codes and thus may underestimate the true rate of falls in an older adult population (10\% from medical records vs. $30 \%$ self-reported). However, there is some evidence that targeting injurious falls is a better strategy for preventing future injurious falls than considering any fall whether injurious or non-injurious [2]. Strengths of the study include a relatively large study population stratified by new and continuing users and numbers of FRD to define suitable at-risk target populations.

\section{Conclusions}

Overall, approximately $44 \%$ of the AARP Medicare Supplement-insured study population used at least one of the FRD . Of these, $29 \%$ were new users and $71 \%$ were continuing users. Fall or hip fracture rates were similar in the two groups. Characteristics associated with the risk of falls included previous injurious fall or hip fracture, use of two or more FRD, older age, poorer health, and being female. The risk of falls demonstrated a dose response increase with increased numbers of FRD . New users of multiple of 
FRD were at highest risk and may be a suitable target for a proactive falls prevention intervention that could include care coordination across multiple prescribers. However, continuing users were also at increased risk of falls, especially with higher numbers of FRD, and they may also benefit from falls awareness and prevention programs.

\section{Compliance with Ethical Standards}

Funding This work was funded by the Medicare Supplement Insurance Program. The research did not receive any specific grant or grant number from funding agencies.

Conflict of interest Shirley Musich, Shaohung Wang, Joann Ruiz and Kevin Hawkins are employed by and hold stock in UnitedHealth Group. Ellen Wicker is employed by AARP Services, Inc. Their compensation was not dependent on the results obtained in this research, and the investigators retained full independence in the conduct of this research.

Ethical approval This research is covered under New England IRB No. 120160532

Open Access This article is distributed under the terms of the Creative Commons Attribution-NonCommercial 4.0 International License (http://creativecommons.org/licenses/by-nc/4.0/), which permits any noncommercial use, distribution, and reproduction in any medium, provided you give appropriate credit to the original author(s) and the source, provide a link to the Creative Commons license, and indicate if changes were made.

\section{References}

1. Centers for Disease Control and Prevention (CDC). Fatalities and injuries from falls among older adults—United States, 1993-2003 and 2001-2005. MMWR Morb Mortal Wkly Rep. 2006;55:1221-4.

2. Pohl P, Nordin E, Lundquist A, et al. Community-dwelling older people with an injurious fall are likely to sustain new injurious falls within 5 years: a prospective long-term follow-up study. BMC Geriatr. 2014; 14:120.

3. French DD, Werner DC, Campbell RR, et al. A multivariate fall risk assessment model for VHA nursing homes using the minimum data set. J Am Med Dir Assoc. 2007;8:115-22.

4. Grenier S, Payette MC, Langlois F, et al. Depressive symptoms are independently associated with recurrent falls in communitydwelling older adults. Int Psychogeriatr. 2014;23:1-9.

5. Castro VM, McCoy TH, Cagan A, et al. Stratification of risk for hospital admissions for injury. BMJ. 2014;349:g5863.

6. Milos V, Bondesson A, Magnusson M, et al. Fall risk-increasing drugs and falls: a cross-sectional study among elderly patients in primary care. BMC Geriatr. 2014;14:40-7.

7. Sjöberg C, Bladh L, Klintberg L, et al. Treatment with fall-riskincreasing and fracture-preventing drugs before and after hip fracture. Drugs Aging. 2010;27:653-61.

8. Sjöberg C, Wallerstedt SM. Effects of medication reviews performed by a physician on treatment with fracture-preventing and fall-risk-increasing drugs in older adults with hip fracture-a randomized controlled study. J Am Geriatr Soc. 2013;6:1464-72.

9. Kragh A, Elmståhl S, Atroshi I. Older adults' medication use 6 months before and after hip fracture: a population-based cohort study. J Am Geriatr Soc. 2011;59:863-8.
10. van der Velde N, Stricker BH, Pols HA, et al. Risk of falls after withdrawal of fall-risk-increasing drugs: a prospective cohort study. Br J Clin Pharmacol. 2007;63:232-7.

11. Ham AC, Swart KM, Enneman AW, et al. Medication-related fall incidents in an older, ambulant population: the B-PROOF study. Drugs Aging. 2014;31:917-27.

12. Bennett A, Gnjidic D, Gillett M, et al. Prevalence and impact of fall-risk-increasing drugs, polypharmacy, and drug-drug interactions to robust versus frail hospitalized falls patients: a prospective cohort study. Drugs Aging. 2014;31:225-32.

13. Boyé ND, van der Velde N, de Vries OJ, et al. Effectiveness of medication withdrawal in older fallers: results from the Improving Medication Prescribing to reduce Risk Of FALLs (IMPROveFALL) trial. Age Ageing. 2017;46(1):142-6.

14. French DD, Campbell R, Spehar A, et al. Outpatient medications and hip fractures in the US. Drugs Aging. 2005;22:877-85.

15. French DD, Campbell R, Spehar A, et al. Drugs and falls in community-dwelling older people: a national veterans study. Clin Ther. 2006;28:619-30.

16. Woolcott JC, Richardson KJ, Wiens MO, et al. Meta-analysis of the impact of 9 medication on falls in elderly persons. Arch Intern Med. 2009;169:1952-60.

17. Huang AR, Mallet L, Rochefort CM, et al. Medication-related falls in the elderly. Drugs Aging. 2012;29:359-76.

18. Munson JC, Bynum JP, Bell JE, et al. Patterns of prescription drug use before and after a fragility fracture. JAMA Intern Med. 2016;176:1531-8.

19. Weiner DK, Hanlon JT, Studenski SA. Effects of central nervous system polypharmacy on falls liability in community-dwelling elderly. Gerontology. 1998;44:217-21.

20. National Committee for Quality Assurance. HEDIS ${ }^{\circledR}$ measures included in the 2016 Quality Rating System (QRS). http://www. ncqa.org/hedis-quality-measurement/hedis-measures/hedis-2016. Accessed 17 Dec 2016.

21. Hill KD, Wee R. Psychotropic drug-induced falls in older people. Drugs Aging. 2012;29:15-30.

22. Berdot S, Bertrand M, Dartigues JF, et al. Inappropriate medication use and risk of falls-a prospective study in a large community-dwelling elderly cohort. BMC Geriatr. 2009;9:30.

23. Wagner AK, Zhang F, Soumerai SB, et al. Benzodiazepine use and hip fractures in the elderly: who is at greatest risk? Arch Intern Med. 2004;164:1567-72.

24. Neutel CI, Perry S, Maxwell C. Medication use and risk of falls. Pharmacoepidemiol Drug Saf. 2002;11:97-104.

25. Maxwell CJ, Neutel CI, Hirdes JP. A prospective study of falls after benzodiazepine use: a comparison of new and repeat use. Pharmacoepidemiol Drug Saf. 1997;6:27-35.

26. Mott DA, Martin B, Breslow R, et al. Impact of a medication therapy management intervention targeting medications associated with falling: results of a pilot study. J Am Pharm Assoc. 2016;56:22-8.

27. Campbell AJ, Robertson MC, Gardner MM, et al. Psychotropic medication withdrawal and a home-based exercise program to prevent falls: a randomized, controlled trial. J Am Geriatr Soc. 1999;47:850-3.

28. Kaiser Family Foundation. Medigap: spotlight on enrollment, premiums, and recent trends. April 2013. https:// kaiserfamilyfoundation.files.wordpress.com/2013/04/8412-2.pdf. Accessed 14 Dec 2016.

29. Pope GC, Kautter J, Ingber MJ, et al. Evaluation of the CMSHCC risk adjustment model. 2011 http://www.cms.gov/ Medicare/Health-Plans/MedicareAdvtgSpecRateStats/ downloads/evaluation_risk_adj_model_2011.pdf. Accessed 14 Dec 2016. 
30. Charlson ME, Pompei P, Ales KL, et al. A new method of classifying prognostic comorbidity in longitudinal studies: development and validation. J Chronic Dis. 1987;40:373-83.

31. National Committee for Quality Assurance. Improving specialist care coordination. http://www.ncqa.org/Portals/0/Public\% 20Policy/2013\%20PDFS/Improving_Specialty_Care_

Coordination.pdf. Accessed 19 Dec 2016.

32. Bell HT, Steinsbekk A, Granas AG. Factors influencing prescribing of fall-risk-increasing drugs to the elderly: a qualitative study. Scand J Prim Health Care. 2015;33:107-14.
33. Gillespie LD, Robertson MC, Gillespie WJ, et al. Interventions for preventing falls in older people living in the community. Cochrane Database Syst Rev. 2012;9:CD007146.

34. Gawler S, Skelton DA, Dinan-Young S, et al. Reducing falls among older people in general practice: The ProAct65+ exercise intervention trial. Arch Gerontol Geriatr. 2016;67:46-54.

35. Lee SH, Kim HS. Exercise interventions for preventing falls among older people in care facilities: ameta-analysis. Worldviews Evid Based Nurs. 2017;14(1):74-80. 